Inula britannica (non L.) quoad pl. ex Japon.-Matsumura, l. e. p. 651. (1912).

Inula britannica subsp. japonica KrTAMURA, in litt.

Nom. Jap. Oguruma, Yezo-oguruma.

Hab. at the foot of Mt. Apoi, Samani, etc. Fl. Aug.

Dist. Kyushu, Shikoku, Honshu, Yezo, Korea, China and Manchuria.

$602^{(40) * I n u l a ~ s a l i c i n a ~ L i n n a E u s, ~ S p . ~ P l . ~ e d . ~ 1, ~ I I, ~ p . ~} 882(1753)$.

* var. asiatica Kitamura in Acta Phytotax. et Geobot. II, p. 44 (1933).

Syn. Inula salicina (non L.) quoad pl. ex Japon.-Matsumura, Ind. Pl. Jap. II-2, p. 652 (1912).-TATEw., Veg. Apoi p. 114 (1928).

Inula involucrata (non Kaleniczenkow 1845) Miquel in Amm. Mus.

Bot. Lugd.-Bat. II, p. 171 (1866).-NAKaI, Veg. A poi p. 72 (1930).

Nom. Jap. Kasensô.

Hab. . at the foot of Mt. Apoi, Samani, etc. Fl. Aug.

Dist. Kyushu, Shikoku, Honshu, Yezo, Korea and Manchuria.

(To be continued.)

\title{
Contributions to the Knowledge of the Systematics of Morus in Japan. IX.
}

\section{Morus in Shikoku and Kiusiu.}

By

\section{Teikichi Hotta"'.}

With a Text-figures.

Received October 28, 1937.

Various papers have hitherto been reported on Morus in Shikoku and

1) Deep acknowledgement is due to Prof. Emer. K. Mryabe and Prof. S. Ito, who have given constant guidance, to Prof. Y. Tochinar and Di. M. Tateivaki, who have rendered kind advices, to Prof. T. NAkai, Dr. M. Ionda, Prof. G. Koidzumi and Dr. R. TAKenouchi, who helped the writer in many ways, as well as to those authorities of the Forestry Bureau and other institutions in Shikoku and Kiusiu, who were kind enough to send specimens at his request. 
Kiusiu by G. Kordzumi ${ }^{2)}$, Y. Dor ${ }^{3)}$, G. Masamưne ${ }^{4)}$ and S. Hatsushim. ${ }^{5)}$. They have, however, dealt with Morus bombycis KoIDz. and its variety caudatifolia KoIDz., Morus tiliaefolia Makino and Morus australis Poner.

\section{Key to the species, subspecies, varieties and forms of Morus found in Shikoku and Kiusiu.}

1. Style long, stigma divided into two parts at its apex.

Sect. I. Dolichostylae KoIDz, ......... 2

Style none or very short with sessile or subsessile stigma divided into two parts.

Seet. II. Macromorus KoIDz. . . ........13 Morus tiliaefolia MAKINo.

2. Cystolith of epidermis is rather acute at apex, rarely erooked. Apex of the leaf abruptly acuminate or acuminate. ................ bombycis Koldz......3 Cystolith of epidermis is usually projecting at the upper part, and obtuse at apex. Apex of the leaf caudate or long acuminate. .............. australis PoIrers.

3. Upper surface of the leaf scabrous, or rarely smooth, and both surfaces hirsute on the veins, margin irregularly dentato-serrate or rarely dentato-erenate.

M. bombycis KoIDz., var. typica НоттА .....4 Upper surface of the leaf smooth and both surfaces glabrous, margin dentatoserrulate, veins and petiole very slender.

M. bombycis KoIDz., var. Koketsuana HotrA.

4. Leaf lolsate or not lobate and ovate or ovato-elliptical. $\ldots \ldots \ldots \ldots \ldots \ldots \ldots$ Leaf testudiniform. ................... testudiniformis Horra.

5. Both surface with a few short hairs, rarely hairless only on the under surface. . 6 Upper surface of the leaf hirsute on the veins, under surface with rather dense hairs. var. pubescens Hotra.

6. Apex of the leaf eaudate or subulate. $\ldots \ldots \ldots \ldots \ldots \ldots \ldots \ldots \ldots \ldots \ldots \ldots$ Apex of the leaf abruptly acuminate or acuminate or acute. $\ldots \ldots \ldots \ldots \ldots \ldots$

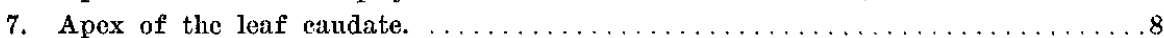

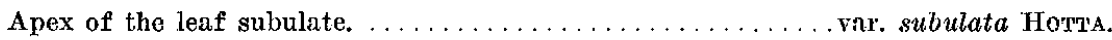

8. Leaf $2-4(-6)$ lobate or not lobate. . . . . . . var. caudatifolia Kordz. . . . .9 Leaf irregularly doubly lobate, upper surface asperate or very scabrous.

var. pseudodiabolica Нотта.

9. Upper surface of the leaf seabrous or somewhat smooth. . . . . . . . . . 10 Upper surface of the leaf very asperate rarely scabrous, apex caudate or long

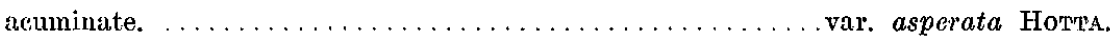

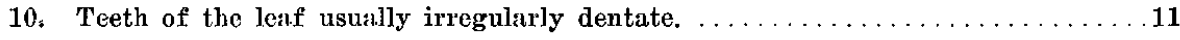
Teeth of the leaf very irregularly dentate, leaf cordate in shape.

var. irregularidentata HonTs.

11. Juanf lobate or not lobate. In the former ease usually the length of the lobe is

2) KoIdzumr, G.:--Synopsis Specierum Generis Mori, in Bull. Imp. Serieult. Exp. Stat., II, 1, p. 10, 14, 19 (1923).

3) Dor, Y.:-Florula Satsumensis sive Enumeratio Plantarum in Provincia Satsuma, Sponte Nascentium, I, 3, p. 30 (1928).

4) MaSAMUne, G.:-Moraceae in Floristic and Geobotanical Studies on the Tsland of Yakushima, Prov. Ôsumi, in Mem. Fac. Se. Agrieult. Tatihoku Imp. Univ., XI, (1934).

5) Intsushima, S.:-Moraceae in Report Experimental Forest Faculty Agriculture, Kiusiu Imp. Univ., 5, p. 54-5 (1934). 
nbout half-way between the margin and midrib. f. normalis HoтrA. Leaf is pinnatisect or pinnatifid and the segments are very narrow.

f. dissecta NAKAI.

Sect. I. Dolichostylae KoIdzumi, Imp. Sericult. Exp. Stat., II, 1, p. 3 (1923).

(1) Morus bombycis Kordzumi, Bot. Mag. (Tokyo), XXIX, p. 313 (1915).

Nom. Jap. Yamaguwa.

Nom. Vern. Marulkuwa, Mimikire-guwa.

Hab.

Shikoku.

Prov. Sanuki : Mt. Konpira (T. HotTa, no. 10125).

Prov. Awa: Shinryo, Myosaigun (R. Uchinara, no. 25026) ; Sakaki, Yokose (Z. Nozaki, no. 25028) ; Mt. Takagoshi (Z. Tashino, fr. \&, ex herb. Univ. Kyo.) ; Soyayama, Mimagun (no. 2564, ex herb. Univ. Tok.) ; Shishikui, Kaibugun (K. Kawaseye, no. 25025) ; Ogawa, Higashisoyayama, Mimagun (S. KaneKo, no. 25002); Mitsuna (T. Hotta, no. 10571).

Prov. Iyo: Koyayama, Mikawa, Kamiukenagun (Z. Sugiyama, no. 39097 ) ; Mt. Aina, Omokawa, Kamiukena (M. Yamanaka, no. 39050); Mt. Koyayama, Mikawa, Kamiukenagun (Z. Sugryama, no. 39096) ; Kigatsutoge, Higashiuwagun ('T. HotтA, no. 10567) ; Osu (T. HоттA, no. 10716); Mt. Deishi (T. HotтA, no. 10577) ; Mt. Hobashiraya, Goso, Minamiuwagun (M. Sugaftara, no. 39093) ; Mt. Sumiyoshi, Uwajima (T. Hotra, no. 10230); Ogawa, Iyayama, Mimagun (S. Kaneko, no. 25020).

Prov. Tosa: Takeyashiki, Kitagawa, Akigun (K. HARA, no. 10968); Osugi (T. Hotta, no. 10115 et 30426); Suzaki (T. Hotta, no. 10128); Motokawa, Tosagun (S. Kuhara, no. 30431 et Y. OKabaYAShr, no. 30418); Ikegawa, Azumagun (S. Kawakam.I, no. 30417) ; Mt. Miyanotani, Taishyomura (S. FuJII, no. 110-A).

Kiusiu.

Prov. Chikuzen: Oshima (S. Takasu et K. Hanada, no. 11456, fl. \&, ex herb. Univ. Kyu.) ; Nishitozaki (M. Takenouchi, no. 865, fl. \&, ex herb. Univ. Kyu.); Insula Genkai, Kitazaki, Itoharagun (K. NaKamura, no. 476) ; Hakozaki (T. Ho're, no. 10082) ; Mt. Yufu (S. Hanada, no. 13549, ex herb. Univ. Kyu.) ; Misetoge (M. TAKenoHCHI, no. 3256, ex herb. Univ. Kyu.) ; Gokayama, Minamihata, Chikushigun (S. SAтo, no. 30134).

Prov. Buzen: Yabakei (M. Takenouchi, no. 6215, ex herb. Univ. Kyu.).

Prov. Chikugo: Tomarisako, Hasegawa, Onogun (I. KaI, no. 30357) ; Maezaki (M. TAkenouchi, no. 864, fl. $\hat{o}$, ex herb. Univ. Kyu.) ; Kuroki (T. Ho'tTa, no. 39077) ; Yoshii ('T. HotTA, no. 10569) ; 'Toyama ('T. HotтA, 
39066 ) ; Mt. Yosomuki (T. Horta, no. 10121) ; Ginsui, Miikegun (T. Sugıno, ex herb. Univ. Kyo., ?).

Prov. Bungo: Morimachi, Kusugun (Y. Kato, no. 39885) ; Yutsubo, Iida, Kusugun (K. Iто, nо. 39882) ; Tano, Iida, Kusugun (S. Іто, no. 39877); Nogami, Kusugun (Y. Kato, no. 39880); Minaniyamada, Kusugun (S. Iтo, no. 39879) ; Yuhira (T. HotTA, no. 10056); Otake, Naworigun (M. KukU, no. 30355) ; Kusumi, Naorigun (B. Furudera, no. 30353) ; Sayeki (F. Takasu, no. 39156) ; Kawaragi, Minamiamabegun (G. TAKaGr, no. 39159) ; Nagano, Kodachi, Minamiamabegun (F. TAKasU, no. 39163) ; Nawomi (T. HoTre, no. 9138).

Prov. Tsushima: Kotomura, Kamigatagun (S. SuyedA, no. 30159) ; Mt. Tatsura (Z. Tashiro, ex herb. Univ. Kyu., ?) ; Zusan, Shimogatagun (S. Kuroki, no. 30162).

Prov. Hizen: Matsukuma, Higashiseburi, Kanzakigun (S. SATo, no. 30120 ) ; Matsukuma, Higashiseburi, Kanzakigun (S. SAto, no. 30132) ; Kitayama, Ogigun (S. KogA, no. 30133) ; Shimokumagawa, Minamiyamamura, Ogigun (M. Moriu, no. 30123) ; Matsuo, Ogigun (N. TaJima, no. 30122); Minamiyama, Ogigun (S. Yamaguchi, no. 30119) ; Aichi, Higashimatsuuragun (K. ICHINo, no. 30127) ; Yamashiro, Higashimatsuuragun (K. YoroYAMA, no. 39104); Hisasato, Higashimatsuuragun (S. IKI); Ike, Aichi, Higashimatsuuragun (K. Icnino, no. 30130) ; Kurimura, Higashimatsuuragum (K. ICHINo, no. 30128); Kagamimura, Higashimatsuuragun (Y. IrIGUCHI, no. 30121) ; Imari, Nishimatsuuragun (E. Minamisako, no. 39129); Takikawa, Higashiyamashiro, Nishimatsuuragun (K. Yокоу ама, no. 39128); Higashimachi, Minamimatsuuragun (I. Hramoto, no. 30180); Arikawa, Minamimatsuuragun (I. Hrramoto, no. 30182) ; Shimabara (M. Takenouchr, no. 863, ex herb. Univ. Kyu., ?) ; Nokomi, Fujitsugun (R. Kawasaki, no. 39095) ; Nanaura, Fujitsugun (S. SaKaI, no. 39089) ; 'Tanoye, Matsuosato, Tomiguchi (Y. Miyazaki, no. 744-A) ; Kishuku, Minamimatsuuraguu (I. IIIramóto, no. 30414).

Prov. Higo: Sakashita (T. Horta, no. 39062) ; Uehimaki ('T. HoTta, no. 25006, typus) ; Seta ('T. Hotra, no. 55004) ; Takamori (R. KoKeTsu, K. Hanada et M. Takenouchi, ex herb. Univ. Kyu.) ; Momikimura, Yatsushirogum (M. Takenouchi, no. 4738, ex herb. Univ. Kyu.); Shimomatsukuma, Yatsushirogun (T. HotTa, no. 9163) ; Sakamoto (T. HoTTA, no. 413) ; Aida, Tamagun (M. Uchiyama, no. 39075) ; Yamaji, Aida, Tamagun (G. KoInz., fl. + , ex herb. Univ. Kyo.); Watari, Tamagun (S. Fukuyama, no. 39085 ).

Prov. Hiuga: Yamada, Kitamorogatagun (N. TAKenoucri, no. 39144) ; Minamigo, Iigashiusukigun (A. IchuriarA, no. 25006) ; Minamigo, Yamada, Kitamorogatagun (N. TAKEnouchI, no. 39142) ; Nagaseko, Sanzai, Koyugun (K. Shiraishi, no. 594-A); Nagaseko, Sanzai, Koyug'un (T. НоттA, no. 
30252) ; Yonera (K. Mayebara, no. 542, fr. $\$$, ex herb. Univ. Kyo.) ; Kawakita, Tono, Koyugun (H. Shinohara, no. 30416) ; Takanabe, Koyugun ( $\mathrm{T}$. Hotta, no. 379) ; Sanno, Koyugun (Y. TAguchi et S. KaI, no. 30251) ; Sadohara, Koyugun (T. НоттA, no. 939) ; Uchiyama, Takaoka, Higashimorogatagun (S. TAKAHASHI, no. 30379); Uchiyama Takaoka, Higashimorogatagun (S. Takahashi, no. 30370); Ikume, Miyosakigun (T. Hotta, no. 9158); Shiramizu, Hokita, Kamihokita (H. SHIGA, no. 30359) ; Hokita, Kamihokita (H. Shiga, no. 30363) ; Kawakami, Hokita, Kamihokita (T. Murakami, no. 25016 ) ; Nasaki, Suki Nishimorogatagun (Y. Hond, no. 30384); Shyomeiji, Masaki, Nishimorogatagun (M. Uchiуama, no. 39079); Uchino, Masaki, Nishimorogatagun (M. Uchry ama, no. 39078) ; Sakiyama, Takasaki, Kitamorogatagun (N. TakenouchI, no. 39140) ; Mt. Kirishima, Nishidake, Kitamorogatagun (N. TAKENOUCHI, no. 39136) ; Sakiyama, Takasaki, Kitamorogatagun (N. TAKenouchi, no. 39148) ; Fukushima ('T. НотTA, no. 39052).

Prov. Osumi: Shimoyaku, Kumakegun (M. Axamatsu, no. 30268); Tagami, Tarumizu, Kimotsukigun (I. INo, no. 30129) ; Takakuma, Kanoya, Kimotsukigun (T. Hoтta, no. 198) ; Kushira (T. HotтA, no. 197) ; Oaira ('T. Hotta, no. 5980); Fumoto, Tashiro (I. NIIHo, no. 19134).

Prov. Satsuma: Awoki, Oguchi, Isagun (M. Uchiyama, no. 39049); Kuna, Satsumagun (K. Asoda, no. 30437); Kawauchi, Satsumagun (T. Iwashita, no. 30118) ; Ibusuki, Ibusukigun (T. HotTA, no. 744-B, cult. ?).

Distr. Sachalin, australis insula Tontomushiri (ex Kordz. op.) Hokkaido, Honshiu, Korea.

var. Koketsuana HоттA, var. nov.

Frutex; cortice fusca. Folium subcordatum vel ovato-oblongum, valde herbaceum, supra laeve, utrinque fere glabrum, apice acuminato vel subito, acuminato, margine dentato-serrulata, basi truncata vel aperte cordata, venis valde gracilis; petiolo gracile $2-2.5 \mathrm{~cm}$. longo.

Nom. Jap. Hosori-guwa (nov.).

Hab.

Prov. Higo: Aso (R. Kokersu et K. Hanada, no. 7464, ex herb. Univ. Kyu.).

Remarks. Leaf is ovato-oblong and very thin, upper surface is smooth, both surfaces are rather glabrous, acuminate or abruptly acuminate at the apex, margin dentato-serrulate, truncate or shallowly cordate at the base, vein is very slender; petiole very slender and $2-2.5 \mathrm{~cm}$. in length.

var. testudiniformis Нотта, var. nov.

Folium varie profunde lobatum fere testudiniforme, apice longicaudato vel subulato, subtricuspidato, basi truncata vel aperte cordata. 
Nom. Jap. Kameba-guwa (nov.).

Hab.

Kiusiu. Prov. Hizen : Honmachi, Minamimatsuuragun (I. Hirano, no. 30181, typus). Prov. Hiuga: Sakiyama, Takasaki, Kitamorogatagun (N. Takenouchi, no. 39141). Prov. Satsuma: Oaira, Kimotsukigun (T. Ho'tTa, no. 5980 ).

Distr. Honshiu.

var. pubescens HotтA, Trans. Sapporo Nat. Hist. Soc., XIV, 3, p. 201, t. VI, e (1936) et XIV, 4, p. 274 (1936), (New to Shikoku et Kiushiu).

Syn. Morus japonica AUdin, var. pubescens Yendo, Traité sur la Cult. dı̀ Mûr. au Jap., p. 17 (1930) nom. seminud.

Ramulus plerumque tenuis, fere declinatus. Folium subtus insigniter pubescens.

Nom. Jap. Keyama-guwa (nov.).

Hab.

Shikoku. Prov. Tosa: Motokawa, Tosagun (I. Kuhara, no. 39238).

Kiusiu. Prov. Bungo: Nogami, Kuzu (Y. KAKU, no. 39883, typus). Prov. Hiuga: Nasaki, Sukimura (Y. Honda, no. 30383). Prov. Osumi: Awa, Shimoyaku (M. Akamatsu, no. 30266).

Distr. Yezo, Honshiu.

var. subulata Hотта, Bot. Mag. (Tokyo), LI, 608, p. 692 (1937).

Apex folii subulatus rare caudatus, basi cuneata vel truncata aperte cordata.

Nom. Jap. Kirisaki-yamaguwa.

Hab.

Shikoku. Prov. Tosa: Yekawasaki, Hantagun (S. FuJII, no. 39972).

Kiusiu. Prov. Hiuga: Shomioji, Shinkomura (M. Uсhimura, no. 39084-A, typus).

Distr. Honshiu et in hortis eulta.

var. pseudodiabolica HotTA, var. nov.

Folium irregulariter duplo-lobatum, subtus fere pubescens, supra asperatum vel valde scabrum, apice caudato; margine fere arguti-serrnlata vel dentato-serrata, apice apiculato; petiolo omnino pubescente.

Nom. Jap. Onagaoni-guwa (nov.).

Hab.

Shikoku. Prov. Tosa: Yekawasaki, Hanta (S. FuJII, no. 39975, typus); Suhara, Takaokagun (S. FuJII, no. 108-A).

Remarks. Leaf is irregularly doubly lobate, upper surface of the leaf are asperate or very scabrous, under surface hirsute on the veins. Apex 
is caudate, margin is slightly arguto-dentate or dentato-serrate, the apices of the teeth are apiculate, petiole with rather conspicuously white hairs on the whole surface.

var. caudatifolia KoIDzumi, Kuwazoku shokubutsu Kô, p. 33 in nota sub $M$. bombycis (1919) ; Bull. Sericult. Exp. Stat., II, 1, p. 12 (1923).NakaI, Fl. Sylv. Korea, XIX, p. 102, t. XXXII (1932).-HortTa, Phytotax. Geobot., VI, 2, p. 110 (1937).

Syn. Morus caudatifolia Kordzumi, Matsumura, Ieon. Pl. Koishikawaensis III, 4, p. 97, Pl. 185 (1916).-Makino et NEmoto, Fl. Jap., p. 1085 (1925).

Folium: apice caudato subito elongato.

Nom. Jap. Onaga-guwa.

Hab.

Shikoku. Prov. Tosa: Suhara, Takaoka (S. FuJI, no. 108-A).

Kiusiu. Prov. Chikugo: Fukushima ('T. Ho'rtA, no. 39052). Prov. Bungo: Higashiiida, Kuzugun (K. Yoshikawa, no. 39881, typus). Prov. Hiuga: Minamisato, Higashiusuki (A. Ichrнara, no. 25003); Nishidake, Kitamorogatagun (N. TAKENOUCHI, no. 39136).

Distr. Hokkaido, Honsiu et Korea.

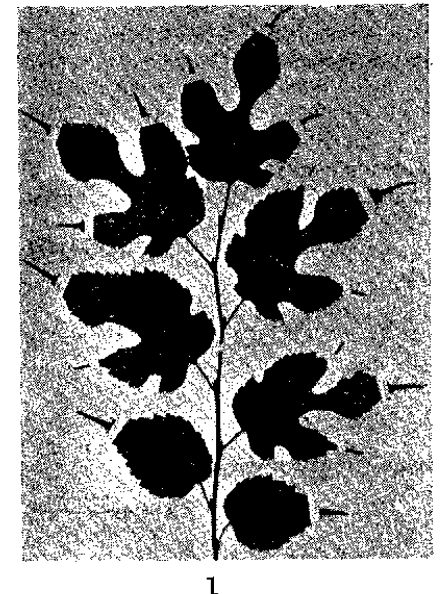

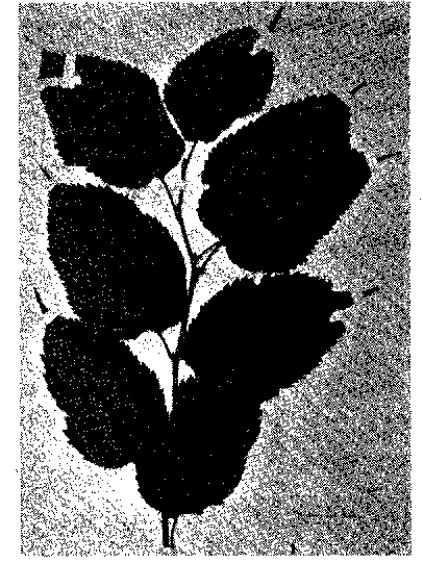

2

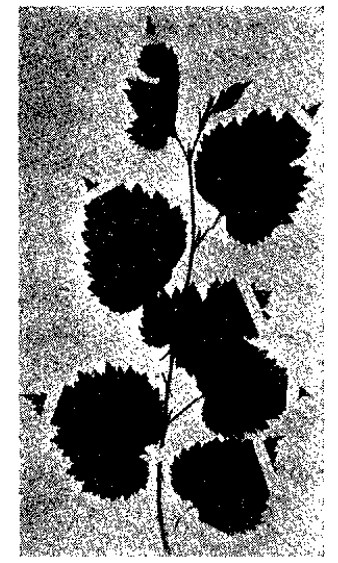

3

Fig. 1. M. bombycis Kordz., var. caudatifolia KoIDz.

Fig. 2. M. bombycis KoIDz., var. asperata HoTTA,

Fig. 3. M. bombycis KoIDz., var. irregularidentata НоттА.

var. asperata Нотта, var. nov.

Folium supra valde asperatum rare scaberrimum, apice caudato vel longiacuminato. 
Nov. Jap. Zaraba-guwa (nov.).

Hab.

Shikoku. Prov. Tosa: Motokawa, Tosagun (U. Окамото, no. 30421).

Kiusiu. Prov. Bungo: Higashiiida, Kuzugun (K. YoshrKawa, no. 39884, typus). Prov. Hiuga: Sakiyama, Takasaki (N. Takenouchi, no. 39147). Prov. Osumi : Kanoya (S. Yezono, no. 30194).

Distr. Honshiu.

var. irregularidentata Нотта, var. nov.

Folium cordatum, apice plerumque tricuspidato, margine volde irreguralidentata, basi plerumque asperte cordata.

Nom. Jap. Midarehanguwa (nov.).

Nom. Verm. Kashiba.

Hab.

Kiusiu. Prov. Satsuma: Yamano, Isagun(M. Hyodo, no. 29942, typus).

Distr. Honshiu.

form. dissecta NakaI, Fl. Sylv. Korea., XIX, p. 101, t. 29 B (1932).HotтA, Trans. Sapporo Nat. Hist. Soc. XIV, 3, p. 203 (1936) ; Bot. Mag. (Tokyo), LI, 611, p. 838 (1937). (New to Shikoku et Kiusiu).

Syn. Morus alba var. stylosa form. dissecta NAKAI, Veget. Isl. Quelpaert, p. 39, no. 5176 (1914), nom. nud.

Folium pinnatifidum vel bipinnatifidum, lobis angustis.

Nom. Jap. Hosoba-yamaguwa.

Hab.

Shikoku. Prov. Awa: Sakaki, Yokose (Z. Nozaki, no. 25030). Prov. Iyo: Mt. Odafuka, Ukenagun (Z. Sugryama, no. 39054); Mt. Sumiyoshi, Uwajima (T. Hotra, no. 10534). Prov. Tosa: Mt. Miyanotani, Taishyomura (S. Fuji no. 105-A) ; Sakashima, Taisyomura (S. FuJII, no. 104-A).

Kiusiu. Prov. Bungo: Amagase (T. HotTA, no. 10079). Prov. Hizen: Mt. Nunofusa (T.Sumita, no. 10057). Prov. Higo: Hazama, Aita, Tamagun (M. Uchiyama, no. 39076). Prov. Hiuga: Nishigo, Higashiusukigun ('T'. Ura, no. 25015) ; Tono, Koyugum (S. Yamane, no. 25013) ; Sadohara, Koyugun (T. Нотта, no. 408); Uchiyama, Takaoka, Higashimorogatagun (S. TAKahashi, no. 30368) ; Kirishima, Nishidake, Kitamorogatagun (N. TAKEnouchi, no. 39151). Prov. Satsuma : Tarumizu, Kimotsukigun (T. Нотта, no. 30196, typus) ; Yamano, Isagun (M. Ochial, no. 39943).

Distr. Yezo, Honsiu, Shikoku, Kiusiu, Korea.

(2) Morus australis POIRET.

Morus australis PoIret, Lamarck Ency. Method. Bot., IV, p. 380(1797).

Nom. Jap. Shima-guwa. 
Hab.

Kiusiu. Prov. Osumi : Higashiamagi (S. Үокота, no. 39020).

Remarks: Cystolith of epidermis is usually projecting at the upper part, and obtuse at apex.

Distr. in region trop. et subtrop. Asia, Australis.

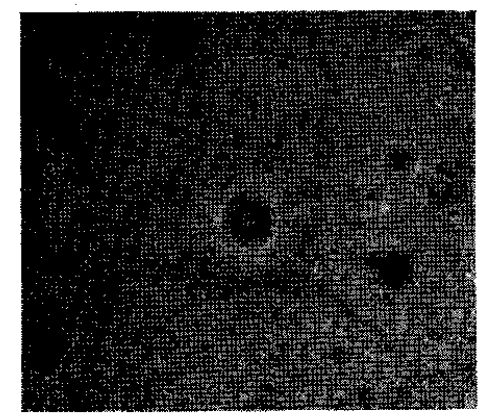

4

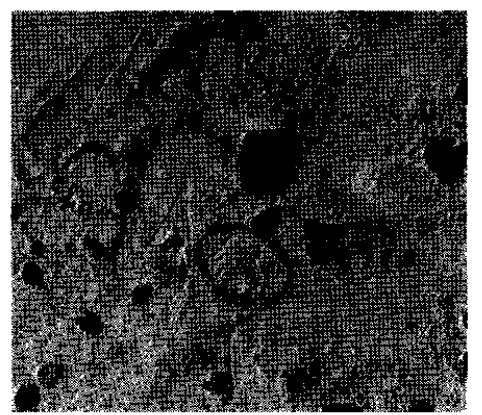

อ

Fig. 4. Cystolith of a leaf of $M$. australis PoIRET.

Fig. 5. Cystolith of a leaf of $M$. tiliaefolia Makino. Zeiss C $\times$ Leitz Periplan $8(\times 180)$.

Sect. II. Macromorus KoIDzumI, Imp. Sericult. Exp. Stat., II, 1, p. 4 (1923).

(3) Morus tiliaefolia Makino.

Morus tiliaefolia MaKrno, Bot. Mag. (Tokyo), XXIII, p. 88 (1909). Nom. Jap. Keguwa.

Hab.

S̃ilikoku. Prov. Awa: Komimachi, Mimagun (G. KordzumI, ex Herb. Univ. Kyo.). Kiusiu. Prov. Tsushima: Nii (T. NakaI, ex Herb. Univ. Tok.).

Remarks. Cystolith of epidermis is broad ovate with rather conicopapillate, and acute tip.

Distr. Honsiu, Korea. 